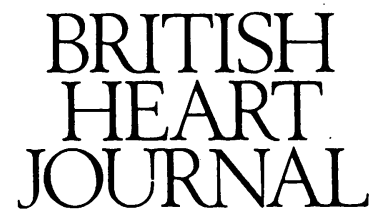

Editorial

\title{
Hypertrophic cardiomyopathy: one disease or several?
}

The first clinical or pathological descriptions of a disease are inevitably biased by patient selection. With time the range of features encompassed by the disease widens inevitably leading to a consideration of where the actual limits lie-some wish to "split" others to "lump". Hypertrophic cardiomyopathy has reached this stage and it is now uncertain whether there is one genetic abnormality with protean manifestations or several genetic abnormalities, all of which produce morphological and clinical features of abnormal ventricular hypertrophy.

Teare provided the first unequivocal illustration of the morphological features of what is now known as hypertrophic cardiomyopathy. ${ }^{1}$ His paper is the most cited paper from the British Heart Journal: it has been quoted more than 390 times. $^{2}$ His method of dissecting the heart was unorthodox but had the advantage of clearly displaying the anatomy of the left ventricular outflow tract. Thus he was able to recognise that there was an association between asymmetrical hypertrophy of the interventricular septum, bulging out beneath the aortic valve, and sudden unexpected death in young individuals. Coincidental with this morphological description there was growing recognition of subaortic stenosis in living individuals, and it was not difficult to see that the pathological features described by Teare were the likely basis for this.

Teare also described the characteristic histological appearances of bundles of myocytes running in several directions and separated by connective tissue. The presence of a typical specimen of asymmetrical septal hypertrophy, misclassified as a rhabdomyoma of the heart in St George's Medical School museum, biased him towards the idea of a localised benign tumour and he therefore did not appreciate that the biological changes represented a generalised abnormality of heart muscle.

During the 1970 s it became recognised that the disease was not as circumscribed in its clinical features as the name asymmetrical septal hypertrophy implied. Echocardiographic surveys showed that while the upper septum and anterior wall of the left ventricle was the most common site of hypertrophy the segment of abnormal muscle may lie in the mid-septal, lateral, posterior, or apical regions of the left ventricle ${ }^{34}$ or in the right ventricle. ${ }^{5}$ Symmetrical involvement of the left ventricle is another recognised variant. ${ }^{6}$ Family studies show that the expression of the disease within a family is very variable. ${ }^{7}$ Teare later recognised that the morphological features of the disease were more varied than his initial description of asymmetrical septal hypertrophy and that it occurred in elderly people dying of other causes. ${ }^{8}$ The early descriptions of the disease emphasised the high risk of both sudden death and cardiac failure, but later surveys pointed out that the disease can be relatively benign and compatible with a long asymptomatic phase. ${ }^{79}$ Anatomical criteria have now been used to create a broader definition of hypertrophic cardiomyopathy as a hypertrophied, non-dilated, left ventricle in the absence of a cardiac or systemic disease that itself could produce left ventricular hypertrophy. ${ }^{10}$

Ideally there should be a single "gold standard" for the disease-the histological features seem to be an obvious candidate. One characteristic of hypertrophic cardiomyopathy, unlike hypertrophy secondary to an increased volume or pressure load, is the very uneven increase in myocyte size and nuclear ploidy. Another characteristic, the myocyte disorganisation or disarray first recognised by Teare, has a variety of forms. Myofibrillar disorganisation is an intracellular abnormality of contractile filament organisation, and its specifity is low because it is also found in a wide range of cardiomyopathies. True disarray is a malarrangement of cell to cell contact-the shape of individual myocytes is abnormal and lateral attachments are increased. The histological pictures are complex and depend on the plane in which the cells are sectioned. ${ }^{11}$ The most common form consists of areas of myocardium in which adjacent cardiac muscle cells are aligned obliquely, producing a whorled, tangled, or pinwheel configuration. This histological appearance, however, is not absolutely specific because it is also found in focal areas in normal hearts as well as in hearts in which valve disease has resulted in hypertrophy. ${ }^{12}$ The junction of the right ventricular free wall and the septum is especially likely to have focal areas of disarray. But the extent of disarray has a high specificity and sensitivity for hypertrophic cardiomyopathy. Maron and Roberts used a planimetric method to measure the percentage area of the septum occupied by disarray in 54 patients with a firm clinical and echocardiographic diagnosis of hypertrophic cardiomyopathy. ${ }^{113}$ They used 144 hearts from normal individuals or from those with hypertrophy caused by aortic valve disease, hypertension, and congenital heart disease for comparison. The mean (SEM) percentage area of septum containing disarray was 31 (3)\% in hypertrophic cardiomyopathy and $1.5(0.6) \%$ in controls. Whereas a cut off point of $5 \%$ gives a $86 \%$ sensitivity and $90 \%$ specificity for disarray being diagnostic of hypertrophic cardiomyopathy, six patients with all the other hallmarks of hypertrophic cardiomyopathy had less than 5\% disarray and three of these had no disarray at all.

Two control hearts showed more than $20 \%$ disarray. As a corollary to this demonstration of the variable amount and uneven distribution of disarray in whole hearts, biopsy in life can neither confirm nor refute the diagnosis of hypertrophic cardiomyopathy. The chance of obtaining false negative findings is high because of sampling error; false positives arise because the biopsy specimen can include a focal area of disarray in a heart without hypertrophic cardiomyopathy. This does not mean that the use of a scoring system based on a constellation of abnormal histological findings in a biopsy specimen including the myocyte size, nuclear size, and myocyte disorganisation cannot distinguish between hypertrophic cardiomyopathy 
and normal hearts ${ }^{14}$ but only that biopsy will not resolve a doubtful clinical diagnosis.

The number of myocytes in a transect of the septum was higher in hypertrophic cardiomyopathy than when an equivalent degree of hypertrophy was attributable to a known cause such as hypertension. ${ }^{15-17}$ Such results indicate that in hypertrophic cardiomyopathy there is an increase in the total number of myocytes (hyperplasia) as well as an increase in myocyte size (hypertrophy). It is not known whether this hyperplasia occurs in utero, in early childhood, or in the adolescent growth phase. Sequential echocardiographic study throughout life in individuals from families with hypertrophic cardiomyopathy suggests that segments of hypertrophic myocardium are first detected in the adolescent growth phase and do not subsequently spread out into the rest of the ventricle. ${ }^{18}$

Two papers in this issue of the British Heart Journal propose a further widening of the clinical spectrum of hypertrophic cardiomyopathy. Maron et al (p 308) describe a young man, from a family known to have hypertrophic cardiomyopathy, who dropped dead: necropsy showed a macroscopically normal heart of normal weight and no hypertrophy. ${ }^{19}$ Histological examination, however, showed extensive myocardial disarray. The implication is that had the patient not died the typical echocardiographic abnormality of segmental hypertrophy would have appeared later in life. A further possibility is shown in the paper by McKenna et al (p 287) who describe two families with a clear history of sudden death. ${ }^{20}$ At necropsy none of the victims, some of whom were adults, showed evidence of hypertrophy as assessed by left ventricular mass or shape but the percentage of myocyte disarray was well within the range characteristic of hypertrophic cardiomyopathy. These papers show that it is possible to have cases which have good cause to be considered as hypertrophic cardiomyopathy but do not actually have hypertrophy when this is defined as an increase in left ventricular mass or thickness.

The question remains whether there is one genetic abnormality encompassing all these different morphological and clinical expressions or whether there are several genetic abnormalities with a final common pathway of abnormal myocardial hypertrophy.

Identification of the gene or genes responsible would resolve the issue. A start has been made to identify by linkage studies the chromosomes responsible in affected families. ${ }^{21}$ A site on chromosome 14 was identified in three families with considerable certainty. The genes for the heavy chains of cardiac myosin are on this chromosome. Further linkage studies will establish whether all affected families are the same and the relation of hypertrophic cardiomyopathy to conditions such as neurofibromatosis, Friedreich's ataxia, and Noonan's syndrome-all of which can cause a cardiac disease morphologically similar to hypertrophic cardiomyopathy. Initial results have identified one family with typical hypertrophic cardio- myopathy in which there is no linkage to a locus on chromosome 14 (W J McKenna, 1990, personal communication). This implies that at least two separate gene abnormalities can produce the disease known as hypertrophic cardiomyopathy.

British Heart Foundation

M J DAVIES

Cardiovascular Pathology Unit

St George's Hospital Medical School,

Cranmer Terrace,

London SW17 ORE

1 Teare $\mathrm{D}$. Asymmetrical hypertrophy of the heart in young adults. Br Heart J 1958;20:1-8.

2 Davies MJ. First demonstration of septal hypertrophic cardiomyopathy. Current Contents Life Science 1989;32:17.

3 Maron BJ, Gottdiener JS, Bonow RO, Epstein SE. Hypertrophic cardiomyopathy with unusual locations of left ventricular hypertrophy
undetectable by $M$-mode echocardiography. Circulation 1981;63:409-18.

4 Wigle ED, Sasson Z, Henderson MA, et al. Hypertrophic cardiomyopathy: the importance of the site and extent of hypertrophy. A review. Prog the importance of the site and
Cardiovasc Dis 1985;28:1-83.

5 Rakowski H, Sasson Z, Liu P, Wigle ED. Various patterns of left ventricular hypertrophy in hypertrophic cardiomyopathy. In: Toshima H, Maron BJ eds. Hypertrophic cardiomyopathy. Cardiomyopathy update 2. Tokyo University of Tokyo Press, 1988:283-93.

6 Shapiro LM, McKenna WJ. Distribution of left ventricular hypertrophy in hypertrophic cardiomyopathy: a two-dimensional echocardiographic study. J Am Coll Cardiol 1983;2:437-44.

7 Maron BJ, Nicholas PF, Pickle LW, Wesley YE, Mulvihill JJ. Patterns of inheritance in hypertrophic cardiomyopathy: assessment by $\mathbf{M}$-mode and two-dimensional echocardiography. Am J Cardiol 1984;53:1087-94.

8 Davies MJ, Pomerance A, Teare RD. Pathological features of hypertrophic obstructive cardiomyopathy. J Clin Pathol 1974;27:529-35.

9 Spirito P, Chiarella F, Carratino L, Berisso MZ, Bellotti P, Vecchio C. Clinical course and prognosis of hypertrophic cardiomyopathy in an Clinical course and prognosis of hypertrophic cardiomy
outpatient population. N Engl J Med 1989;320:749-54.

10 Maron BJ, Epstein SE. Recent observations regarding the specificity of three hallmarks of the disease: asymmetric septal hypertrophy, septal disorganhallmarks of the disease: asymmetric septal hypertrophy, septal disorganisation and systolic ante
Cardiol 1980;45:141-54.

11 Maron BJ, Roberts WC. Quantitative analysis of cardiac muscle cell disorganisation in the ventricular septum of patients with hypertrophic cardiomyopathy. Circulation 1979;4:689-706.

12 Becker AE, Caruso G. Myocardial disarray. A critical review. Br Heart J 1982;47:527-38.

13 Maron BJ, Anan TJ, Roberts WC. Quantitative analysis of the distribution of cardiac muscle cell disorganisation in the left ventricular wall of patients with hypertrophic cardiomyopathy. Circulation 1981;63:882-94.

14 Sekiguchi $M$, Nunoda $S$, Hiroe $M$. The value of endomyocardial biopsy in diagnosing hypertrophic cardiomyopathy. In: Toshima H, Maron BJ, eds. Hypertrophic cardiomyopathy: Cardiomyopathy update 2. Tokyo: Univer-
sity of Tokyo Press, 1988:59-72.

15 Fujiwara $H$, Fujiwara $T$, Hamashima $Y$, Kawai C. Number and size of myocytes, amount of interstitial space and extent of disarray of the hearts in patients with systemic hypertension and asymmetric septal hyperin patients with systemic hypertens
trophy. Jpn Circ $J 1985 ; 49: 40414$.

16 Unverferth DV, Baker PB, Pearce LI, Lautman J, Roberts WC. Regional myocyte hypertrophy and increased interstitial myocardial fibrosis in hypertrophic cardiomyopathy. Am J Cardiol 1987;59:932-6.

17 Frenzel H, Schwartzkopff B, Reinecke P, Kamoni K, Losse B. Evidence for muscle fiber hyperplasia in the septum of patients with hypertrophic obstructive cardiomyopathy. Quantitative examination of endomyocardial biopsies and myectomy specimens. $Z$ Kardiol 1987;76(suppl 3):14-9.

18 Maron BJ, Spirito P, Wesley Y, Arce J. Development in and progression of left ventricular hypertrophy in children with hypertrophic cardiomyopathy. N Engl J Med 1986;315:610-4.

19 Maron BJ, Kragel AH, Roberts WC. Sudden death in hypertrophic cardiomyopathy with normal left ventricular mass. Br Heart 1990;63:308-10.

20 McKenna WJ, Stewart JT, Nihoyannopoulos P, McGinty F, Davies MJ. Hypertrophic cardiomyopathy without hypertrophy: two families with Hypertrophic cardiomyopathy without hypertrophy: two families with
myocardial disarray in the absence of increased myocardial mass. Br Heart myocardial disarray in
$J$ 1990;63:287-90.

21 Jarcho JA, McKenna W, Pare JAP, et al. Mapping a gene for familial hypertrophic cardiomyopathy to chromosome 14q1. N Engl $J$ Med 1989;321:1372-7. 